\title{
A VORTICITY-DIVERGENCE GLOBAL SEMI-LAGRANGIAN SPECTRAL MODEL FOR THE SHALLOW WATER EQUATIONS
}

November 29, 2001

Prepared by

John B. Drake

Daniel X. Guo 


\section{DOCUMENT AVAILABILITY}

Reports produced after January 1, 1996, are generally available free via the U.S. Department of Energy (DOE) Information Bridge:

Web Site: http://www.osti.gov/bridge

Reports produced before January 1, 1996, may be purchased by members of the public from the following source:

National Technical Information Service

5285 Port Royal Road

Springfield, VA 22161

Telephone: 703-605-6000 (1-800-553-6847)

TDD: $703-487-4639$

Fax: 703-605-6900

E-mail: info@ntis.fedworld.gov

Web site: http://www.ntis.gov/support/ordernowabout.htm

Reoports are avilable to DOE employees, DOE contractors, Energy Technology Data Exchange (ETDE), and International Nuclear Inforamtion System (INIS) representatives from the following sources:

Office of Scientific and Technical Information

P.O. Box 62

Oak Ridge, TN 37831

Telephone: $865-576-8401$

Fax: 865-576-5728

E-mail: reports@adonis.osti.gov

Web site: http://www.osti.gov/contact.html

This report was prepared as an account of work sponsored by an agency of the United States Government. Neither the United States nor any agency thereof, nor any of their employees, makes any warranty, express or implied, or assumes any legal liability or responsibility for the accuracy, completeness, or usefulness of any information, apparatus, product, or process disclosed, or represents that its use would not infringe privately owned rights. Reference herein to any specific commercial product, process, or service by trade name, trademark, manufacturer, or otherwise, does not necessarily constitute or imply its endorsement, recommendation, or favoring by the United States Government or any agency thereof. The views and opinions of authors expressed herein do not necessarily state or reflect those of the United States Government or any agency thereof. 
ORNL/TM-2001/216

\title{
A VORTICITY-DIVERGENCE GLOBAL SEMI-LAGRANGIAN SPECTRAL MODEL FOR THE SHALLOW WATER EQUATIONS
}

\author{
John B. Drake \\ Computer Science and Mathematics Division \\ Oak Ridge National Laboratory \\ Oak Ridge, Tennessee \\ Daniel X. Guo \\ Department of Mathematics and Statistics \\ University of North Carolina at Wilmington \\ Wilmington, North Carolina
}

Date Published: November 29, 2001

Prepared by

OAK RIDGE NATIONAL LABORATORY

Oak Ridge, Tennessee 37831-6285

managed by

UT-Battelle, LLC

for the

U.S. DEPARTMENT OF ENERGY

under contract DE-AC05-00OR22725 



\section{Contents}

LIST OF FIGURES v v

ACKNOWLEDGMENTS vii

1 INTRODUCTION 1

2 SHALLOW WATER EQUATIONS IN SPHERICAL GEOMETRY 3

3 VORTICITY AND DIVERGENCE FORMS OF THE SHALLOW-WATER EQUATIONS $\mathbf{5}$

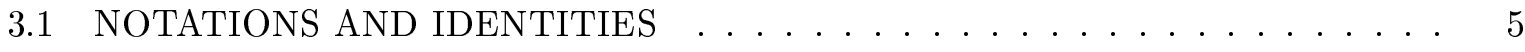

3.2 VORTICITY FORM . . . . . . . . . . . . . . . . . . . . 7

3.3 DIVERGENCE FORM $\ldots \ldots \ldots \ldots \ldots \ldots \ldots$

4 SEMI-IMPLICIT, SEMI-LAGRANGIAN FORMULATION 9

$5 \quad$ SEMI-LAGRANGIAN INTERPOLATIONS $\quad 13$

6 SPECTRAL SEMI-IMPLICIT FORMULATION $\quad 17$

7 NUMERICAL EXPERIMENTS AND RESULTS 21

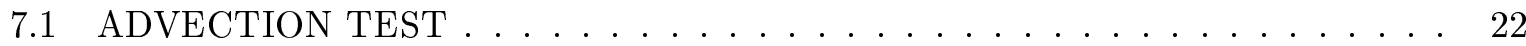

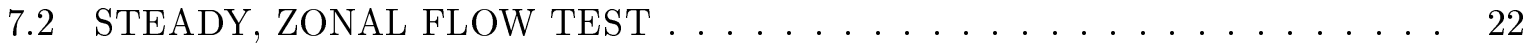

7.3 STEADY STATE ZONAL FLOW WITH COMPACT SUPPORT $\ldots \ldots \ldots$

7.4 ZONAL FLOW OVER AN ISOLATED MOUNTAIN . . . . . . . . . . . . . . . . . 24

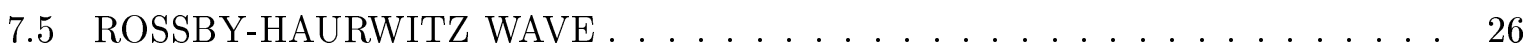

8 CONCLUSIONS $\quad 31$

$\begin{array}{ll}\text { REFERENCES } & 33\end{array}$ 



\section{List of Figures}

1 Standard T42 mesh viewed from $(330,10) \ldots \ldots \ldots \ldots \ldots$

2 Case 1 Contour plots of the height field for $\alpha=\pi / 2-0.05$ and $\Delta t=1200$ seconds. (a) Initial field; (b) after one rotation (12 days); (c) Height field error; (d) $l_{1}$ (solid), $l_{2}$ (short dash), and $l_{\infty}$ (long dash) errors. Contour interval is $100 \mathrm{~m}$ for the field and $2.5 \mathrm{~m}$ for the error. . . . . . . . . . . . . . .

3 Case 2 (a) Contour plot of the height field error for $\alpha=\pi / 2-0.05$ and $\Delta t=1200$ seconds. Contour interval is $0.05 \mathrm{~m}$. (b) $l_{1}$ (solid), $l_{2}$ (short dash), and $l_{\infty}$ (long dash) errors. . . . . . . . . . . . . . . . . . . . . 24

4 Case 3 (a) Contour plot of the height field error after 5 days, for $\alpha=\pi / 2-0.05$ and $\Delta t=1200$ seconds; (b) $l_{1}$ (solid), $l_{2}$ (short dash), and $l_{\infty}$ (long dash) errors. 25

5 Case 5 The difference in height field from initial condition after one day for

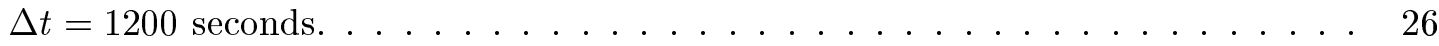

6 Case 5 (cont.) Contour plots of the height field for $\Delta t=1200$ seconds at (a) day 0 , (b) day 5, (c) day 10 , and (d) day 15 on a cylindrical equidistant projection.

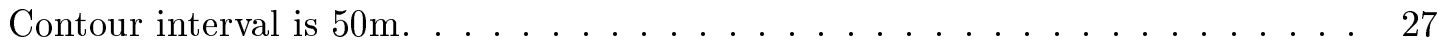

7 Case 5 (cont.) Relative change of global mass (solid), relative change of total energy (short dash), and enstrophy (long dash) . . . . . . . . . . . . 28

8 Case 6 Contour plots of the height field for $\Delta t=1200$ seconds at (a) day 0 , (b)

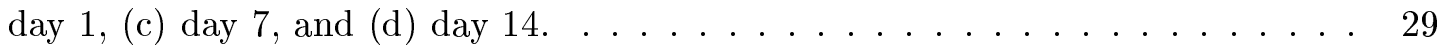

9 Case 6 (cont.) Change in global mass (solid), total energy (short dash), and enstrophy $($ long dash $) \ldots \ldots \ldots$. . . . . . . . . . . . . . 30 



\section{ACKNOWLEDGEMENTS}

This research was supported by the Climate Change Prediction Program of the Atmospheric and Climate Research Division, Office of Energy Research, U.S. Department of Energy under contract DE-AC05-00OR22725 with UT-Battelle, LLC. Computing resources were provided by the Center for Computational Science-Climate and Carbon Research at Oak Ridge National Laboratory. 



\section{INTRODUCTION}

The shallow water equations modeling flow on a sphere are useful for the development and testing of numerical algorithms for atmospheric climate and weather models. A new formulation of the shallow water equations is derived which exhibits an advective form for the vorticity and divergence. This form is particularly well suited for numerical computations using a semiLagrangian spectral discretization. A set of test problems, standard for the shallow water equations on a sphere, are solved and results compared with an Eulerian spectral model.

The semi-Lagrangian transport method was introduced into atmospheric modeling by Robert, Henderson, and Turnbull [6]. A formulation based on a three time level integration scheme in conjunction with a finite difference spatial discretization was studied by Ritchie [4]. Two time level grid point schemes were derived by Bates et al. [1]. Staniforth and Côté [8] survey developments of the application of semi-Lagrangian transport (SLT) methods for shallow water models and for numerical weather prediction.

The spectral (or spherical harmonic transform) method when combined with a SLT method is particularly effective because it allows for long time steps avoiding the Courant-FriedrichsLewy (CFL) restriction of Eulerian methods, while retaining accurate (spectral) treatment of the spatial derivatives. A semi-implicit, semi-Lagrangian formulation with spectral spatial discretization is very effective because the Helmholz problem arising from the semi-implicit time integration can be solved cheaply in the course of the spherical harmonic transform. The combination of spectral, semi-Lagrangian transport with a semi-implicit time integration schemes was first proposed by Ritchie [5]. A advective formulation using vorticity and divergence was introduced by Williamson and Olson [12]. They introduce the vorticity and divergence after the application of the semi-Lagrangian discretization.

The semi-Lagrangian formulation of Williamson and Olson [12] and Bates et al. [1] has the property that the metric terms of the advective form are treated discretely requiring a delicate spherical vector addition of terms at the departure point and arrival point. In their formulation, the metric terms associated with the advection operator do not appear explicitly. The spherical geometry associated with the combination of vector quantities at arrivial and departure points treats the metric terms and is derived in Bates et al. [1]. The formulation derived in this paper avoids this vector addition. It is possible to do this because our formulation is based entirely on a scalar, advective form of the momentum equations. This new form is made possible by the generalization of a vector identity to spherical geometry. 
In Section 2 the standard form of the shallow water equations in spherical geometry are given. Section 3 presents the vector identities needed to derive an advective form of the vorticity and divergence equations. The semi-implicit time integration and semi-Lagrangian transport method are described in Section 4. The SLT interpolation scheme is described in Section 5. Section 6 completes the development of the discrete model with the description of the semi-implicit spectral equations. A discussion of results on several standard test problems is contained in Section 7 . 


\section{SHALLOW WATER EQUATIONS IN SPHERICAL GEOMETRY}

The shallow water equations in advective form [12] in spherical geometry are

$$
\begin{gathered}
\frac{d \mathbf{v}}{d t}=-f \vec{k} \times \mathbf{v}-\nabla \phi \\
\frac{d \phi^{*}}{d t}=-\phi^{*} \nabla \cdot \mathbf{v}
\end{gathered}
$$

where the vector $\mathbf{v}=u \vec{i}+v \vec{j}$ is the horizontal vector velocity and is always orthogonal to the unit vector $\vec{k}$ in the radial direction. It is assumed that $\bar{i}$ and $\bar{j}$ are two unit vectors in the increasing directions of longitude and latitude respectively.

The free surface geopotential is denoted by $\phi=\phi^{*}+\phi_{s}=g\left(h^{*}+h_{s}\right)$ and $\phi^{*}=\phi^{\prime}+\bar{\phi}$, where $g$ is the gravitational acceleration and $h^{*}$ is the height of the free surface above the bottom height, $h_{s}$. The bottom surface height specifies orography as a time invariant function and $\bar{\phi}$ is the time invariant spatial mean. The Coriolis parameter $f=2 \Omega \sin \theta$ incorporates the effect of rotation of the sphere at latitude $\theta$ with angular velocity $\Omega$. If we consider the global geometry, the equations require no boundary conditions but are posed with initial conditions on $\mathbf{v}$ and $\phi$.

The substantial derivative is given by

$$
\frac{d}{d t}=\frac{\partial}{\partial t}+\mathbf{v} \cdot \nabla
$$

The gradient operator and the term $\nabla \cdot \mathbf{v}$ will be defined later.

Expanding $\phi$ in Eqs. (1) and (2), we obtain

$$
\begin{gathered}
\frac{d \mathbf{v}}{d t}+\nabla \phi^{\prime}=-f \vec{k} \times \mathbf{v}-\nabla \phi_{s} \\
\frac{d \phi^{\prime}}{d t}+\bar{\phi} \nabla \cdot \mathbf{v}=-\phi^{\prime} \nabla \cdot \mathbf{v}
\end{gathered}
$$

For simplicity the primes will be dropped from the equations. 


\section{VORTICITY AND DIVERGENCE FORMS OF THE SHALLOW-WATER EQUATIONS}

In this section an advective form of the vorticity and divergence equations for shallow water will be derived. The derivation of the divergence equation requires that a vector identity be extended to the case of spherical geometry, so the first task is to establish the definitions of the differential operators and present the required identities.

\subsection{NOTATIONS AND IDENTITIES}

In longitude-latitude coordinates $(\lambda, \theta)$ the definitions of gradient, divergence, Laplacian, and curl operators, (denoted respectively by $\operatorname{grad}(\nabla), \operatorname{div}(\nabla \cdot), \nabla^{2}$, and curl) are as follows:

$\operatorname{grad} A: A$ is a scalar function defined on the sphere and

$$
\operatorname{grad} A=\nabla A=\frac{\vec{i}}{a \cos \theta} \frac{\partial A}{\partial \lambda}+\frac{\vec{j}}{a} \frac{\partial A}{\partial \theta}
$$

$\operatorname{div} F: F$ is a tangent vector function $F=F_{1} \bar{i}+F_{2} \bar{j}$ and

$$
\operatorname{div} F=\nabla \cdot F=\frac{1}{a \cos \theta} \frac{\partial F_{1}}{\partial \lambda}+\frac{1}{a \cos \theta} \frac{\partial\left(\cos \theta F_{2}\right)}{\partial \theta}
$$

curl $F: F$ is a tangent vector function as in $\operatorname{div} F$ and

$$
\operatorname{curl} F=\nabla \times F=\frac{\vec{k}}{a \cos \theta}\left[\frac{\partial F_{2}}{\partial \lambda}-\frac{\partial\left(\cos \theta F_{1}\right)}{\partial \theta}\right]
$$

$\nabla^{2} A: A$ is a scalar function defined on the sphere, and we define the Laplacian operator on a scalar function as

$$
\nabla^{2} A=\frac{1}{a^{2} \cos \theta}\left[\frac{\partial}{\partial \lambda}\left(\frac{1}{\cos \theta} \frac{\partial A}{\partial \lambda}\right)+\frac{\partial}{\partial \theta}\left(\cos \theta \frac{\partial A}{\partial \theta}\right)\right] .
$$

In contrast to the Laplacian operator on a scalar function, there are several possible definitions of the Laplacian operator on a vector function. Here we set

$$
\nabla^{2} F=\nabla^{2} F_{1} \bar{i}+\nabla^{2} F_{2} \bar{j}
$$

and use the covariant derivative $\nabla F=\left\{\nabla_{\bar{i}} F, \nabla_{\bar{j}} F\right\}$ of the vector function $F$

$$
\nabla_{\bar{i}} F=\left(\frac{1}{a \cos \theta} \frac{\partial F_{1}}{\partial \lambda}-\frac{F_{2} \sin \theta}{a \cos \theta}\right) \bar{i}+\left(\frac{1}{a \cos \theta} \frac{\partial F_{2}}{\partial \lambda}+\frac{F_{1} \sin \theta}{a \cos \theta}\right) \bar{j}
$$




$$
\nabla_{\bar{j}} F=\frac{1}{a} \frac{\partial F_{1}}{\partial \theta} \bar{i}+\frac{1}{a} \frac{\partial F_{2}}{\partial \theta} \bar{j}
$$

Then we obtain

$$
(\mathbf{v} \cdot \nabla) \mathbf{v}=u \nabla_{\bar{i}} \mathbf{v}+v \nabla_{\bar{j}} \mathbf{v}
$$

To avoid problems with the vector representation of the velocity at the pole and to maintain compatibility with the scalar spectral transform method, we introduce the vorticity $\zeta \equiv \vec{k} \cdot \nabla \times \mathbf{v}$ and the divergence $\delta \equiv \nabla \cdot \mathbf{v}$.

To obtain $\mathbf{v}$ from $\zeta$ and $\delta$, we need another pair of equations. The Helmholtz theorem, which separates the horizontal velocity vector $\mathbf{v}$ into a scalar stream function $\psi$ and a scalar velocity potential $\chi$, states

$$
\mathbf{v}=\vec{k} \times \nabla \psi+\nabla \chi
$$

or in component form

$$
u=\frac{1}{a \cos \theta} \frac{\partial \chi}{\partial \lambda}-\frac{1}{a} \frac{\partial \psi}{\partial \theta}
$$

and

$$
v=\frac{1}{a \cos \theta} \frac{\partial \psi}{\partial \lambda}+\frac{1}{a} \frac{\partial \chi}{\partial \theta}
$$

Application of the curl and divergence operators to Eqs.(5) give the relationships for the prognostic variables $\zeta$ and $\delta$ in terms of the stream function and the velocity potential

$$
\zeta=\nabla^{2} \psi
$$

and

$$
\delta=\nabla^{2} \chi
$$

Using the vector identity

$$
(\mathbf{v} \cdot \nabla) \mathbf{v} \equiv \nabla\left(\frac{\mathbf{v} \cdot \mathbf{v}}{2}\right)+\zeta \vec{k} \times \mathbf{v},
$$

the horizontal momentum equation can be expanded to the form

$$
\frac{\partial \mathbf{v}}{\partial t}+\nabla \phi=-(\zeta+f) \vec{k} \times \mathbf{v}-\nabla\left(\phi_{s}+\frac{\mathbf{v} \cdot \mathbf{v}}{2}\right) .
$$


Also, by direct computation and the definition of the Laplacian operator on a vector function, we obtain

$$
\nabla \times(\nabla \times \mathbf{v})=\nabla(\nabla \cdot \mathbf{v})-\nabla^{2} \mathbf{v}+\frac{1}{a^{2} \cos ^{2} \theta} \mathbf{v}-\frac{2 \sin \theta}{a^{2} \cos ^{2} \theta} \vec{k} \times \frac{\partial \mathbf{v}}{\partial \lambda}
$$

or without the first order derivatives, we rewrite it as

$$
\nabla \times(\nabla \times \mathbf{v})=\nabla(\nabla \cdot \mathbf{v})-\frac{1}{\cos \theta} \nabla^{2}(\mathbf{v} \cos \theta)-\frac{2 \sin \theta}{a \cos \theta} \mathcal{F}
$$

where

$$
\mathcal{F}=\left(\begin{array}{c}
-\zeta \\
\delta
\end{array}\right)
$$

\subsection{VORTICITY FORM}

Applying $\vec{k} \cdot \nabla \times$ to Eqs. (8) gives

$$
\frac{\partial \zeta}{\partial t}=-\vec{k} \cdot \nabla \times[(\zeta+f) \vec{k} \times \mathbf{v}]
$$

Using

$$
\begin{aligned}
\vec{k} \cdot \nabla \times[(\zeta+f) \vec{k} \times \mathbf{v}] & =\nabla \cdot(\zeta+f) \mathbf{v} \\
& =(\zeta+f) \nabla \cdot \mathbf{v}+\mathbf{v} \cdot \nabla(\zeta+f) \\
& =\mathbf{v} \cdot \nabla \zeta+(\zeta+f) \delta+\mathbf{v} \cdot \nabla f
\end{aligned}
$$

we obtain

$$
\frac{\partial \zeta}{\partial t}=-\mathbf{v} \cdot \nabla \zeta-(\zeta+f) \delta-\mathbf{v} \cdot \nabla f
$$

i.e.

$$
\frac{d \zeta}{d t}=-(\zeta+f) \delta-\mathbf{v} \cdot \nabla f
$$

By using $\eta=\zeta+f$, we can rewrite Eqs. (11) as,

$$
\frac{d \eta}{d t}=-\eta \delta
$$

\subsection{DIVERGENCE FORM}

Application of the divergence operator to Eqs. (8) gives

$$
\frac{\partial \delta}{\partial t}+\nabla^{2} \phi=-\nabla \cdot[(\zeta+f) \vec{k} \times \mathbf{v}]-\nabla^{2}\left(\phi_{s}+\frac{\mathbf{v} \cdot \mathbf{v}}{2}\right)
$$


By using the vector identities, we obtain

$$
\begin{aligned}
\nabla \cdot[(\zeta+f) \vec{k} \times \mathbf{v}] & =-\vec{k} \cdot \nabla \times(\zeta+f) \mathbf{v} \\
& =-(\zeta+f) \vec{k} \cdot \nabla \times \mathbf{v}+\vec{k} \cdot \mathbf{v} \times \nabla(\zeta+f) \\
& =-(\zeta+f) \zeta+\vec{k} \cdot \mathbf{v} \times \nabla f+\vec{k} \cdot \mathbf{v} \times \nabla \zeta
\end{aligned}
$$

Notice that

$$
\nabla \zeta=\nabla(\vec{k} \cdot \nabla \times \mathbf{v})=\vec{k} \times[\nabla \times(\nabla \times \mathbf{v})]
$$

From Eqs. (9), we obtain

$$
\nabla \zeta=\vec{k} \times(\nabla \delta-\tilde{\mathcal{F}})
$$

where

$$
\tilde{\mathcal{F}}=\frac{1}{\cos \theta} \nabla^{2}(\mathbf{v} \cos \theta)+\frac{2 \sin \theta}{a \cos \theta} \mathcal{F}
$$

and $\mathcal{F}$ is defined in Eqs. (10). Then,

$$
\begin{aligned}
\vec{k} \cdot \mathbf{v} \times \nabla \zeta & =\vec{k} \cdot \mathbf{v} \times[\vec{k} \times(\nabla \delta-\tilde{\mathcal{F}})] \\
& =\vec{k} \cdot[\mathbf{v} \cdot(\nabla \delta-\tilde{\mathcal{F}})] \vec{k}-\vec{k} \cdot(\mathbf{v} \cdot \vec{k})(\nabla \delta-\tilde{\mathcal{F}}) \\
& =\mathbf{v} \cdot(\nabla \delta-\tilde{\mathcal{F}}) .
\end{aligned}
$$

Combining terms, we obtain,

$$
\frac{\partial \delta}{\partial t}+\nabla^{2} \phi=(\zeta+f) \zeta-\vec{k} \cdot \mathbf{v} \times \nabla f-\mathbf{v} \cdot(\nabla \delta-\tilde{\mathcal{F}})-\nabla^{2}\left(\phi_{s}+\frac{\mathbf{v} \cdot \mathbf{v}}{2}\right)
$$

i.e.

$$
\frac{d \delta}{d t}+\nabla^{2} \phi=(\zeta+f) \zeta-\vec{k} \cdot \mathbf{v} \times \nabla f+\mathbf{v} \cdot \tilde{\mathcal{F}}-\nabla^{2}\left(\phi_{s}+\frac{\mathbf{v} \cdot \mathbf{v}}{2}\right) .
$$

In terms of $\eta$, Eqs. (14) is

$$
\frac{d \delta}{d t}+\nabla^{2} \phi=\eta(\eta-f)-\vec{k} \cdot \mathbf{v} \times \nabla f+\mathbf{v} \cdot \tilde{\mathcal{F}}-\nabla^{2}\left(\phi_{s}+\frac{\mathbf{v} \cdot \mathbf{v}}{2}\right) .
$$

Note that the Eqs. (12) and (15) contain the substantial derivatives of two scalar functions. This form is reminiscent of the forms derived by Truesdell and reported in Serrin [7]. We can apply the semi-Lagrangian method directly to the vorticity and divergence equations to avoid the complications of dealing with operations of the vector functions on the sphere. 


\section{SEMI-IMPLICIT, SEMI-LAGRANGIAN FORMULATION}

A three time level semi-Lagrangian method for the shallow water equations was described in Ritchie [4]. For meteorological models the form of the method is based on a division of terms in the equations between those involved in the fast moving gravity waves, and the slower moving Rossby waves. Let $G$ denote the fast moving wave terms and $R$ the slower wave terms. Then an advected scalar field $F$ is represented by the equation

$$
\frac{d F}{d t}+G=R
$$

Let subscripts ( $)_{A},()_{M}$ and ()$_{D}$ represent quantities evaluated at the arrival point, midpoint and departure point, respectively. Let superscripts $\tau-1, \tau$ and $\tau+1$ represent the time levels at which quantities are evaluated.

Integrating the Eqs. (16) along the trajectory between the departure point and the arrival point

$$
\int_{t^{\tau-1}}^{t^{\tau+1}} \frac{d F}{d t} d t+\int_{t^{\tau-1}}^{t^{\tau+1}} G d t=\int_{t^{\tau-1}}^{t^{\tau+1}} R d t
$$

The first term reduces exactly to

$$
\int_{t^{\tau-1}}^{t^{\tau+1}} \frac{d F}{d t} d t=F_{A}^{\tau+1}-F_{D}^{\tau-1}
$$

while the $G$ and $R$ terms must be approximated using quadrature rules. In this paper we use

$$
\begin{aligned}
& \int_{t^{\tau-1}}^{t^{\tau+1}} R d t=2 \Delta t R_{M}^{\tau}+O\left(\Delta t^{2}\right) \text { midpoint } \\
& \int_{t^{\tau-1}}^{t^{\tau+1}} G d t=\Delta t\left(G_{A}^{\tau+1}+G_{D}^{\tau-1}\right)+O\left(\Delta t^{3}\right) \quad \text { trapezoidal }
\end{aligned}
$$

resulting in the formulation

$$
\frac{F_{A}^{\tau+1}-F_{D}^{\tau-1}}{2 \Delta t}+\frac{G_{A}^{\tau+1}+G_{D}^{\tau-1}}{2}=R_{M}^{\tau}
$$

These three time level, "centered" approximations result in an $O(\Delta t)$ approximation to Eqs. (16); the approximations to $F$ and $G$ are $O\left(\Delta t^{2}\right)$. This approximation is the basis for most 
three level semi-implicit SLT methods. The review article by Staniforth and Côté [8] discusses the development of the SLT method and some of its applications. We note in particular that Ritchie's [4] three level spectral shallow water model was based on this approximation.

We next introduce the spatial average at the midpoint as follows

$$
\frac{F_{A}^{\tau+1}-F_{D}^{\tau-1}}{2 \Delta t}+\frac{G_{A}^{\tau+1}+G_{D}^{\tau-1}}{2}=\frac{R_{A}^{\tau}+R_{D}^{\tau}}{2}
$$

or

$$
F_{A}^{\tau+1}+\Delta t G_{A}^{\tau+1}=F_{D}^{\tau-1}+\Delta t\left[R_{A}^{\tau}+R_{D}^{\tau}-G_{D}^{\tau-1}\right] \equiv N
$$

The spatial averaging has been shown to reduce distortion produced by topographically forced waves while only introducing a further approximation error of $O\left(\Delta x^{2}\right)$ where $\Delta x$ is the length of the trajectory (not the spatial resolution). Since the trajectory length is proportional to the time step $\Delta t$ this is an acceptable error.

The arrival and departure components of $N$ can be defined in terms of the $R$ 's and $G$ 's as

$$
N_{A} \equiv \Delta t R_{A}^{\tau}
$$

and

$$
N_{D} \equiv F_{D}^{\tau-1}+\Delta t\left[R_{D}^{\tau}-G_{D}^{\tau-1}\right]
$$

The assignment of terms of the shallow water equations to $G$ and $R$ results in different numerical algorithms. If we take $G \equiv 0$, then the algorithm is explicit in time. The explicit method requires small timesteps to stabilize the gravity wave components of the solution. A semi-implicit method results by assigning selected terms to $G$, terms that give rise to the gravity waves. In the momentum equation, Eqs. (3), the gravity wave component, $\nabla \phi$, is identified with the implicit $G$ term and the Coriolis term is identified with the explicit term, $R$. We take

$$
G=\left(\begin{array}{c}
0 \\
\nabla^{2} \phi \\
\bar{\phi} \delta
\end{array}\right),
$$




$$
R=\left(\begin{array}{c}
-\eta \delta \\
\eta(\eta-f)-\vec{k} \cdot \mathbf{v} \times \nabla f+\mathbf{v} \cdot \tilde{\mathcal{F}}-\nabla^{2}\left(\phi_{s}+\frac{\mathbf{v} \cdot \mathbf{v}}{2}\right) \\
-\phi \delta
\end{array}\right)
$$

where $\phi+\bar{\phi}+\phi_{s}$ is the free surface geopotential and $\bar{\phi}$ is a constant reference value; $\tilde{\mathcal{F}}$ is defined in Eqs. (13).

The paper will focus on Eqs. (18) with $G$ and $R$ specified by Eqs. (21) and (22). To this end, let $F$ be defined as

$$
F=\left(\begin{array}{c}
\eta \\
\delta \\
\phi
\end{array}\right)
$$

and substitute Eqs. (21) and (22) into (18), to obtain the semi-Lagrangian scheme for the shallow-water model in vorticity-divergence form as

$$
\begin{array}{ll}
\eta_{A}^{\tau+1} & =N_{\eta} \\
\delta_{A}^{\tau+1}+\Delta t\left[\nabla^{2} \phi\right]_{A}^{\tau+1} & =N_{\delta} \\
\phi_{A}^{\tau+1}+\Delta t[\bar{\phi} \delta]_{A}^{\tau+1} & =N_{\phi},
\end{array}
$$

where $N=\left(N_{\eta}, N_{\delta}, N_{\phi}\right)^{T}$ and $N=N_{A}+N_{D}$, the arrival and departure components of $N$, is defined by

$$
N_{A}=\left(\begin{array}{c}
\Delta t[-\eta \delta]_{A}^{\tau} \\
\Delta t\left[\eta(\eta-f)-\vec{k} \cdot \mathbf{v} \times \nabla f+\mathbf{v} \cdot \tilde{\mathcal{F}}-\nabla^{2}\left(\phi_{s}+\frac{\mathbf{v} \cdot \mathbf{v}}{2}\right)\right]_{A}^{\tau} \\
\Delta t[-\phi \delta]_{A}^{\tau}
\end{array}\right)
$$

and

$$
N_{D}=\left(\begin{array}{l}
\eta_{D}^{\tau-1}+\Delta t[-\eta \delta]_{D}^{\tau} \\
\delta_{D}^{\tau-1}+\Delta t\left[\eta(\eta-f)-\vec{k} \cdot \mathbf{v} \times \nabla f+\mathbf{v} \cdot \tilde{\mathcal{F}}-\nabla^{2}\left(\phi_{s}+\frac{\mathbf{v} \cdot \mathbf{v}}{2}\right)\right]_{D}^{\tau}-\Delta t\left[\nabla^{2} \phi\right]_{D}^{\tau-1} \\
\phi_{D}^{\tau-1}+\Delta t[-\phi \delta]_{D}^{\tau}-\Delta t[\bar{\phi} \delta]_{D}^{\tau-1}
\end{array}\right)
$$

There are no first order derivatives involving unknowns, and the only second-order operator 
is the Laplacian. When the spectral transformation is used to discretize the equations, the properties of Laplacian operator will simplify the computation of the solution. 


\section{SEMI-LAGRANGIAN INTERPOLATIONS}

The following quantities in physical space must be interpolated at the departure points: $\mathbf{v}^{\tau}$,

$\eta^{\tau}, \delta^{\tau}, \phi^{\tau}, \eta^{\tau-1}, \delta^{\tau-1}, \phi^{\tau-1}$ and $\Delta(\mathbf{v} \cdot \mathbf{v})^{\tau}, \Delta \phi^{\tau-1}$. With these quantities, $N_{D}$ can be formed. Alternatively, the sums of terms involved in $N_{D}$ can be formed at the grid points and then interpolated to the departure points. This is computationally less expensive and introduces an error only on the order of the interpolation error. The next section, explains how to compute $\Delta(\mathbf{v} \cdot \mathbf{v})^{\tau}$ and $\Delta \phi^{\tau-1}$ by using the spectral properties of the Laplacian operator.

The shape preserving tensor product interpolation scheme developed by Williamson and Rasch [10] is used for all fields involved in the calculation of the $N$ 's. Their scheme is based on a quasi-cubic interpolant.

The departure point calculation integrates the equation

$$
\frac{d \mathbf{x}}{d t}=-\mathbf{v} \text {. }
$$

backwards along the trajectory from the arrival point. A second-order method is used to compute the departure point as follows,

$$
\begin{aligned}
& \lambda_{D}=\lambda_{A}-2 \Delta t u\left(\lambda_{M}, \theta_{M}, t\right) \\
& \theta_{D}=\theta_{A}-2 \Delta t v\left(\lambda_{M}, \theta_{M}, t\right),
\end{aligned}
$$

where $\left(\lambda_{M}, \theta_{M}\right)$ is the midpoint between the arrival point and departure point. The midpoint of the trajectory is calculated by solving

$$
\begin{aligned}
& \lambda_{M}=\lambda_{A}-\Delta t u\left(\lambda_{M}, \theta_{M}, t\right) \\
& \theta_{M}=\theta_{A}-\Delta t v\left(\lambda_{M}, \theta_{M}, t\right),
\end{aligned}
$$

using a fixed point iteration. At each step of this iteration for the midpoint, the velocity field must be interpolated to the current estimate of the midpoint. Previous studies [8] indicate that linear interpolation is adequate for accuracy in the solution of the shallow water equations. A Lagrange cubic was used for interpolation of the velocity field.

To avoid the singularity at the poles and the complexity of interpolation of the velocity field, a three-dimensional technique, studied by Ritchie [5], is used, which projects all two dimensional vectors on the sphere to the three-dimensional vectors in Cartesian coordinates. Let $(X, Y, Z)$ represent the normalized Cartesian coordinates $(x / a, y / a, z / a)$ and $\tilde{u}_{A}$ and $\tilde{v}_{A}$ be the normalized 
velocities

$$
\begin{aligned}
& \tilde{u}_{A}=u\left(\lambda_{A}, \theta_{A}, t\right) / a \\
& \tilde{v}_{A}=v\left(\lambda_{A}, \theta_{A}, t\right) / a .
\end{aligned}
$$

The arrival point and the normalized Cartesian velocity components are

$$
\begin{aligned}
X_{A} & =\cos \lambda_{A} \cos \theta_{A} \\
Y_{A} & =\sin \lambda_{A} \cos \theta_{A} \\
Z_{A} & =\sin \theta_{A},
\end{aligned}
$$

and

$$
\begin{aligned}
& \dot{X}_{A}=-\tilde{u}_{A} \sin \lambda_{A}-\tilde{v}_{A} \cos \lambda_{A} \sin \theta_{A} \\
& \dot{Y}_{A}=\tilde{u}_{A} \cos \lambda_{A}-\tilde{v}_{A} \sin \lambda_{A} \sin \theta_{A} \\
& \dot{Z}_{A}=\tilde{v}_{A} \cos \theta_{A} .
\end{aligned}
$$

We obtain a similar formulation in three dimensions for the calculation of the midpoint

$$
\begin{aligned}
& X_{M}=b_{M}\left(X_{A}-\Delta t \dot{X}_{M}\right) \\
& Y_{M}=b_{M}\left(Y_{A}-\Delta t \dot{Y}_{M}\right) \\
& Z_{M}=b_{M}\left(Z_{A}-\Delta t \dot{Z}_{M}\right),
\end{aligned}
$$

where

$$
b_{M}=\left[1+\Delta t^{2}\left(\dot{X}_{M}^{2}+\dot{Y}_{M}^{2}+\dot{Z}_{M}^{2}\right)-2 \Delta t\left(X_{A} \dot{X}_{M}+Y_{A} \dot{Y}_{M}+Z_{A} \dot{Z}_{M}\right)\right]^{-1 / 2}
$$

and $b_{M}$ ensures that $\left(X_{M}, Y_{M}, Z_{M}\right)$ is on the sphere. The iteration starts by setting $\left(\dot{X}_{M}, \dot{Y}_{M}, \dot{Z}_{M}\right)=$ $\left(\dot{X}_{A}, \dot{Y}_{A}, \dot{Z}_{A}\right)$. After computing $\left(X_{M}, Y_{M}, Z_{M}\right)$, the Cartesian velocity $\left(\dot{X}_{M}, \dot{Y}_{M}, \dot{Z}_{M}\right)$ is interpolated at the midpoint with a Lagrange cubic.

Once the midpoint iteration has converged the departure point is calculated by using a similar equation to (27) as follows,

$$
\begin{aligned}
& X_{D}=X_{A}-2 \Delta t \dot{X}_{M} \\
& Y_{D}=Y_{A}-2 \Delta t \dot{Y}_{M} \\
& Z_{D}=Z_{A}-2 \Delta t \dot{Z}_{M},
\end{aligned}
$$


or by using the symmetry with the arrival point about the midpoint,

$$
\begin{aligned}
& X_{D}=2 b_{D} X_{M}-X_{A} \\
& Y_{D}=2 b_{D} Y_{M}-Y_{A} \\
& Z_{D}=2 b_{D} Z_{M}-Z_{A},
\end{aligned}
$$

where $b_{D}=X_{A} X_{M}+Y_{A} Y_{M}+Z_{A} Z_{M}$.

Notice that Eqs. (31) and (32) are equivalent. For example, let us consider $X_{D}$ only. From Eqs. (30) and (32),

$$
\begin{aligned}
X_{D} & =2 b_{D} b_{M}\left(X_{A}-\Delta t \dot{X}_{M}\right)-X_{A} \\
& =\left(2 b_{D} b_{M}-1\right) X_{A}-2 b_{D} b_{M} \Delta t \dot{X}_{M} \\
& =X_{A}-2 \Delta t \dot{X}_{M}
\end{aligned}
$$

with $b_{D} b_{M}=1$ because $X_{M} \dot{X}_{M}+Y_{M} \dot{Y}_{M}+Z_{M} \dot{Z}_{M}=0$ (multiply (30) by $X_{M}, Y_{M}$ and $Z_{M}$, respectively, and sum).

The departure point calculation is completed by computing the spherical coordinates from (29),

$$
\lambda_{D}=\tan ^{-1}\left(Y_{D} / X_{D}\right) \quad \text { and } \quad \theta_{D}=\sin ^{-1}\left(Z_{D}\right)
$$




\section{SPECTRAL SEMI-IMPLICIT FORMULATION}

The combination of the semi-Lagrangian method with the spectral transform method has proven to be a powerful technique, providing fast solution of the semi-implicit equations and high spatial accuracy. Williamson and Rasch [10] observed that the semi-Lagrangian treatment of the advection eliminates the nonlinear aliasing of terms in the spectral representation. This allows use of more terms of the spectral expansion but using the same physical grid and effectively doubling the resolution at little extra cost.

The spectral transform, or more correctly, the spherical harmonic transform, is based on the representation of scalar fields as a linear combination of spherical harmonics as

$$
\xi(\lambda, \mu)=\sum_{m=-M}^{M} \sum_{n=|m|}^{N(m)} \xi_{n}^{m} P_{n}^{m}(\mu) e^{\mathrm{i} m \lambda}
$$

and

$$
\xi_{n}^{m}=\int_{-1}^{1} \frac{1}{2 \pi} \int_{0}^{2 \pi} \xi(\lambda, \mu) e^{-\mathbf{i} m \lambda} d \lambda P_{n}^{m}(\mu) d \mu,
$$

where $P_{n}^{m}$ are the normalized associated Legendre functions defined as

$$
P_{n}^{m}(\mu)=\sqrt{\frac{(2 n+1)}{2} \frac{(n-m) !}{(n+m) !}}\left(1-\mu^{2}\right)^{\frac{1}{2} m} \frac{d^{m} P_{n}(\mu)}{d \mu^{m}}, \quad \text { for } m \geq 0
$$

and

$$
P_{n}^{m}(\mu)=(-1)^{-m} P_{n}^{-m}(\mu), \quad \text { for } m<0
$$

such that

$$
\int_{-1}^{+1}\left[P_{n}^{m}(\mu)\right]^{2} d \mu=1
$$

and $P_{n}$ are the Legendre polynomials defined by

$$
P_{n}(\mu)=\frac{1}{2^{n} n !} \frac{d^{n}\left(\mu^{2}-1\right)^{n}}{d \mu^{n}}
$$

The latitudinal coordinate varies between $[-1.1]$ with $\mu=\sin \theta$. For all $n$ and $m$, we obtain,

$$
P_{n}^{m}(\mu)=0, \quad \text { if } n<m
$$


(For properties of the associated Legendre functions, the reader is referred to the Appendix B in Washington and Parkinson [9].)

Once spectral coefficients are known, the transformation from spectral to physical space is accomplished using (33) and is usually referred to as harmonic synthesis. The transform from physical space to spectral space (harmonic analysis) uses a discrete version of the continuous transform (34),

$$
\xi^{m}(\mu)=\frac{1}{2 \pi} \int_{0}^{2 \pi} \xi(\lambda, \mu) e^{-\mathbf{i} m \lambda} d \lambda=\frac{1}{I} \sum_{i=1}^{I} \xi\left(\lambda_{i}, \mu\right) e^{-\mathbf{i} m \lambda_{i}}
$$

and

$$
\xi_{n}^{m}=\int_{-1}^{+1} \xi^{m}(\mu) P_{n}^{m}(\mu) d \mu=\sum_{j=1}^{J} \xi^{m}\left(\mu_{j}\right) P_{n}^{m}\left(\mu_{j}\right) w_{j}
$$

where

$$
\lambda_{i}=\frac{2 \pi i}{I}, \quad w_{j}=\frac{2\left(1-\mu_{j}^{2}\right)}{\left[J P_{J-1}\left(\mu_{j}\right)\right]^{2}},
$$

and $\mu_{j}$ denote the Gaussian latitudes. $I$ is the number of gridpoints in the east-west direction. $J$ is the number of Gaussian latitudes from pole to pole. $w_{j}$ is the Gaussian weight at latitude $\mu_{j}$. The Gaussian latitudes $\mu_{j}$ are determinated from the roots of the Legendre polynomial $P_{J}(\mu)$ and, the Gaussian weights satisfy the relationship,

$$
\sum_{j=1}^{J} w_{j}=2
$$

Since the spherical harmonic functions are eigensolutions of the Laplace equation we have

$$
\nabla^{2} P_{n}^{m}(\mu) e^{i m \lambda}=\frac{-n(n+1)}{a^{2}} P_{n}^{m}(\mu) e^{i m \lambda}
$$

Applying the transformation given in Eqs. (34) to each term of Eqs. (24), we will obtain the spectral formulation. Only two types of terms are involved: the terms with constant coefficients and the terms with a Laplacian operator. The spectral representation of Laplacian operator is given by

$$
\Delta \phi=\sum_{m=-M}^{M} \sum_{n=|m|}^{N(m)} \frac{-n(n+1)}{a^{2}} \phi_{n}^{m} P_{n}^{m}(\mu) e^{i m \lambda} .
$$

For simplicity we drop the index $\tau+1$ and the subscript $A$ to obtain the spectral form of 
the governing equations,

$$
\begin{aligned}
\eta_{n}^{m} & =\left\{N_{\eta}\right\}_{n}^{m}, \\
\delta_{n}^{m}+\Delta t C(n) \phi_{n}^{m} & =\left\{N_{\delta}\right\}_{n}^{m}, \\
\phi_{n}^{m}+\Delta t \bar{\phi} \delta_{n}^{m} & =\left\{N_{\phi}\right\}_{n}^{m},
\end{aligned}
$$

where

$$
C(n)=-\frac{n(n+1)}{a^{2}},
$$

and $a$ is the radius of the earth. $\eta_{n}^{m}$ is directly calculated by (41) and the last two equations, (42) and (43), can be solved together for the advanced time level values in spectral space for $\delta_{n}^{m}$ and $\phi_{n}^{m}$. For each mode $(m, n)$ a $2 \times 2$ system of equations needs to be solved,

$$
\left[\begin{array}{cc}
1 & \Delta t C(n) \\
\Delta t \bar{\phi} & 1
\end{array}\right]\left[\begin{array}{c}
\delta_{n}^{m} \\
\phi_{n}^{m}
\end{array}\right]=\left[\begin{array}{c}
\left\{N_{\delta}\right\}_{n}^{m} \\
\left\{N_{\phi}\right\}_{n}^{m}
\end{array}\right]
$$

This is the same system found in the semi-implicit spectral method of Hack and Jakob [2]. The solution can be expressed by an application of Cramer's rule.

With $\eta_{n}^{m}, \delta_{n}^{m}$ and $\phi_{n}^{m}$ at the arrival points at the new time level, the synthesis (inverse spherical harmonic transform) is used to obtain the values of $\phi$ and $\mathbf{v}$ in physical space. Notice that the $\zeta_{n}^{m}$ can be directly calculated from $\eta_{n}^{m}$ at the new time level with the relationship $\eta=\zeta+f$. The components of $\mathbf{v}$ are obtained through the diagnostic relationship in terms of $\eta_{n}^{m}$ and $\delta_{n}^{m}$

$$
U\left(\lambda_{i}, \mu_{j}\right)=-\sum_{m=-M}^{M} \sum_{n=|m|}^{N(m)} \frac{a}{n(n+1)}\left[i m \delta_{n}^{m} P_{n}^{m}\left(\mu_{j}\right)-\zeta_{n}^{m} H_{n}^{m}\left(\mu_{j}\right)\right] e^{i m \lambda_{i}}
$$

and

$$
V\left(\lambda_{i}, \mu_{j}\right)=-\sum_{m=-M}^{M} \sum_{n=|m|}^{N(m)} \frac{a}{n(n+1)}\left[i m \zeta_{n}^{m} P_{n}^{m}\left(\mu_{j}\right)+\delta_{n}^{m} H_{n}^{m}\left(\mu_{j}\right)\right] e^{i m \lambda_{i}}
$$

where

$$
H_{n}^{m}(\mu) \equiv\left(1-\mu^{2}\right) \frac{d P_{n}^{m}(\mu)}{d \mu}
$$


Finally, the velocity field is calculated on physical grid points with

$$
\begin{aligned}
& u\left(\lambda_{i}, \theta_{j}, t\right)=U\left(\lambda_{i}, \mu_{j}\right) / \cos \theta_{j} \\
& v\left(\lambda_{i}, \theta_{j}, t\right)=V\left(\lambda_{i}, \mu_{j}\right) / \cos \theta_{j}
\end{aligned}
$$

and $\eta, \delta$ and $\phi$ are recovered on physical grid point with formula (33). 


\section{NUMERICAL EXPERIMENTS AND RESULTS}

This section presents numerical results of five standard test cases described in Williamson et al. [11]. The cases can be used to characterize the numerical properties of a method and their appropriateness for numerical weather prediction and climate modeling. They provide a means of addressing accuracy as well as quality of solution in terms of conserved quantities. All results are based on the standard T42 mesh as shown in Fig. 1.

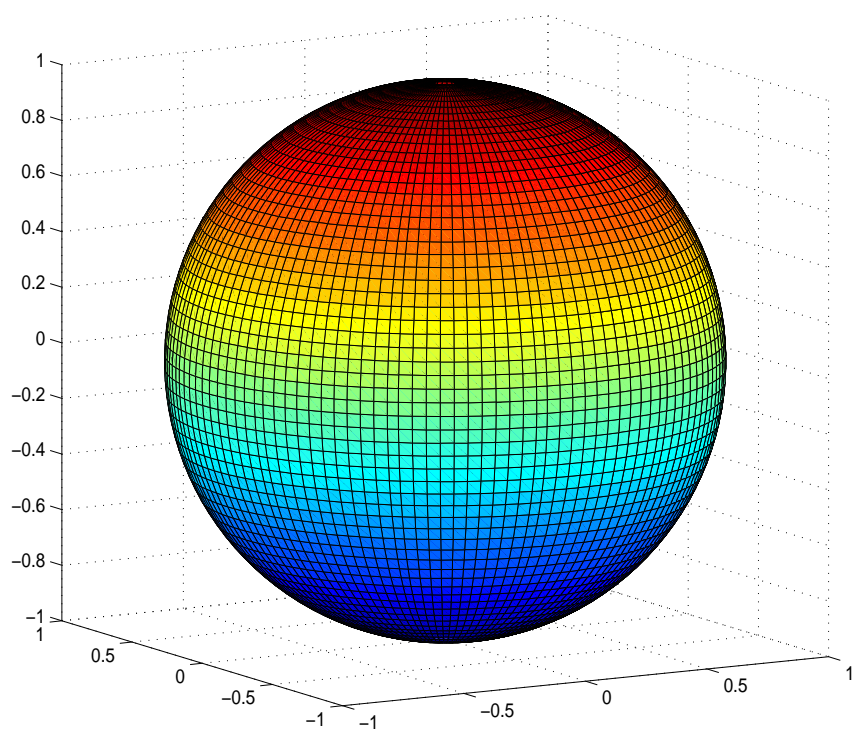

Fig. 1: Standard T42 mesh viewed from $(330,10)$

The discussion will focus on the properties of the solution and the errors in comparison with the T42 Eulerian spectral model. There are four sources of error:

- the truncation error of the time stepping method,

- the error associated with approximation of spatial derivatives,

- the interpolation error of the semi-Lagrangian scheme, and

- the error associated with computing trajectories in the semi-Lagrangian scheme.

It is not always possible to determine which is the dominant error. Information is gained by performing a number of experiments not reported here. For example, the test can be run with several values for the timestep $\Delta t$ to determine if the truncation error is dominant. 


\subsection{ADVECTION TEST}

In this case, we let $\alpha=\pi-0.05$, so the cosine bell is advected over the poles at a slight angle. The initial height field, which should be maintained throughout the course of one rotation, is shown in Fig. 2a. The final height field after one rotation (12 days or 864 time steps of 1200 seconds) is shown in Fig. 2b. Comparing with the initial height field, it is slightly diffused. The difference plot in Fig. 2c illustrates the overall structures of the final height field. It is almost symmetric, but elongated downstream. This is little different from the result of the Eulerian spectral model reported in Jakob et al. [3]. The semi-Lagrangian scheme introduces more dissipation due to the low order cubic interpolation.

The semi-Lagrangian method is relatively insensitive to the time step used. A small timestep is not more accurate leading to the conclusion that the chief source of error is in the semiLagrangian interpolation and the number of times this interpolation is used.

The $l_{1}, l_{2}$, and $l_{\infty}$ error measures are presented in Fig. $2 \mathrm{~d}$. These errors show the same behavior as in Jakob et al. [3], i.e. a significant error associated with the initial representation of the cosine bell followed by a systematic monotonic increase as the cosine bell is advected around the sphere. As the cosine bell crosses the poles at 3 and 9 days, the $l_{1}$ error jumps because of the large meridional grid interval from the missing pole point on the Gaussian grid.

\subsection{STEADY, ZONAL FLOW TEST}

The global zonal geostrophic flow case is a trivial problem for the spectral transform method because the steady solution is exactly representable with a low order spherical harmonic. Errors in the numerical solution from the semi-Lagrangian model reflect the accumulation of rounding errors associated with the SLT cubic interpolation. Figure 3a shows the difference between the analytic solution and the final height field after one rotation (5 days). The $l_{1}, l_{2}$, and $l_{\infty}$ error measures are shown in Fig. 3b. Errors are small and smooth, but monotonically increasing in time.

\subsection{STEADY STATE ZONAL FLOW WITH COMPACT SUPPORT}

The primary difference between this case and the previous case is that the initial condition here is not exactly representable in the spherical harmonic basis. Even so, the errors resulting from the spherical harmonic truncation are small in comparison to the cubic interpolation of the semi-Lagrangian method. Figure 4a shows the difference between the analytic solution and the final height field after one rotation (5 days). The $l_{1}, l_{2}$, and $l_{\infty}$ error measures are shown in 


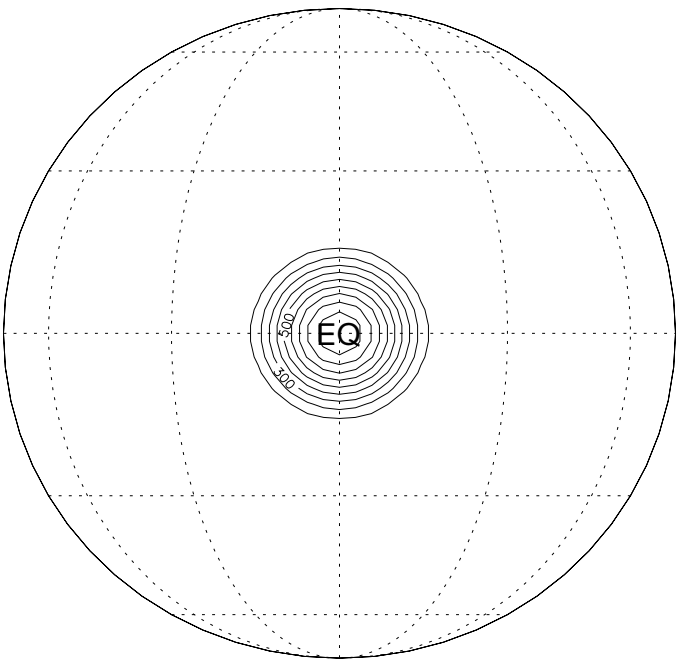

(a)

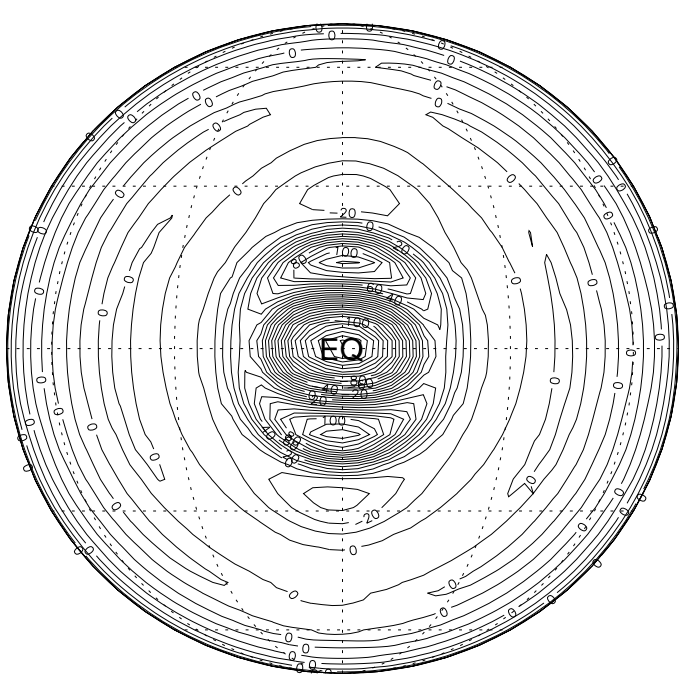

(c)

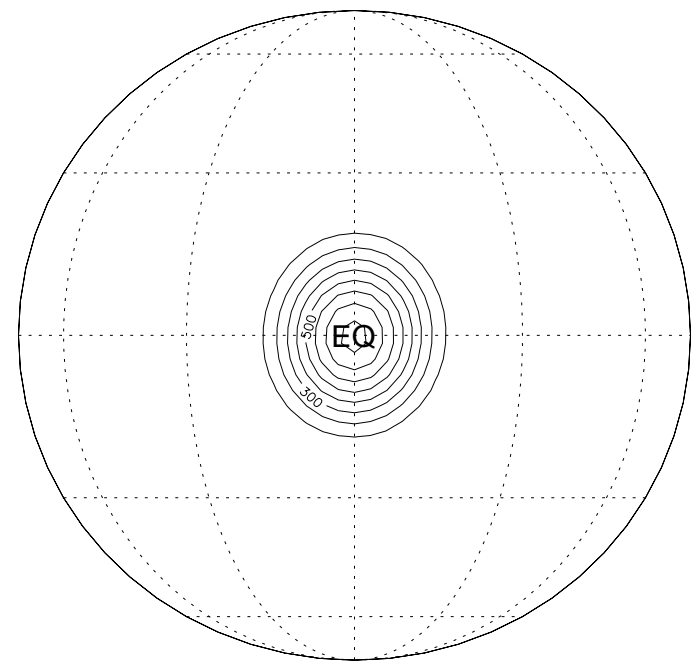

(b)

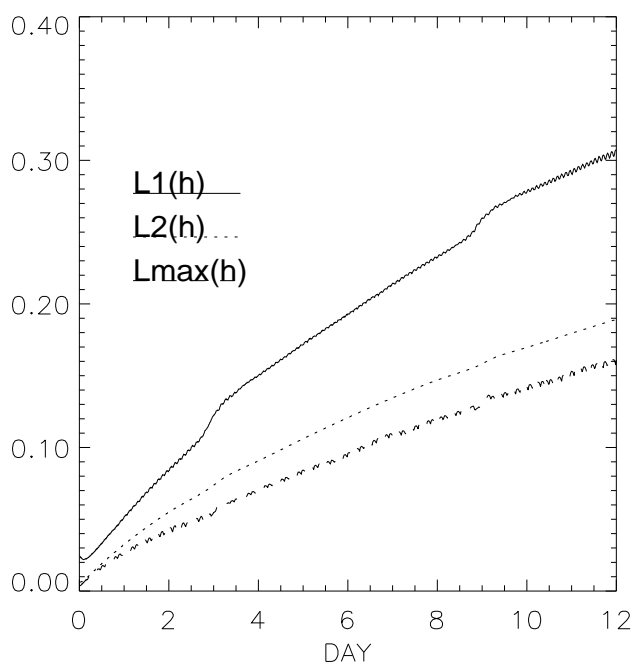

(d)

Fig. 2: Case 1 Contour plots of the height field for $\alpha=\pi / 2-0.05$ and $\Delta t=1200$ seconds. (a) Initial field; (b) after one rotation (12 days); (c) Height field error; (d) $l_{1}$ (solid), $l_{2}$ (short dash), and $l_{\infty}$ (long dash) errors. Contour interval is $100 \mathrm{~m}$ for the field and $2.5 \mathrm{~m}$ for the error. 


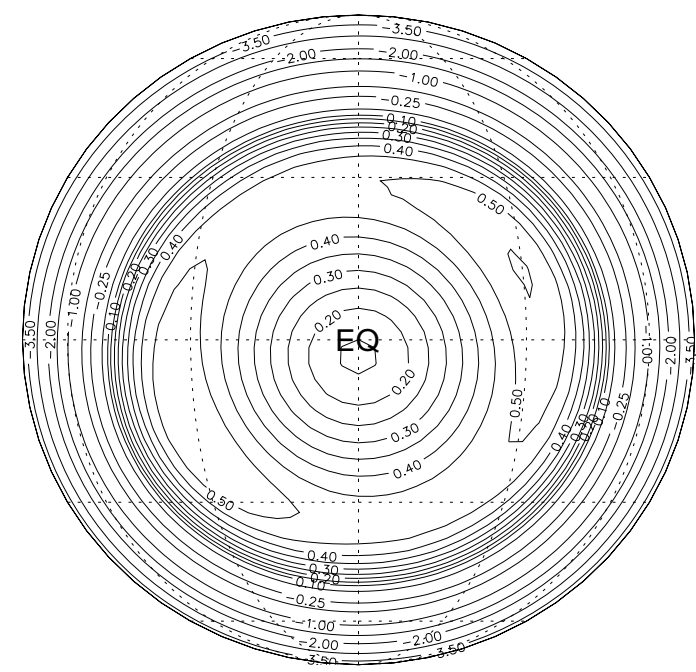

(a)

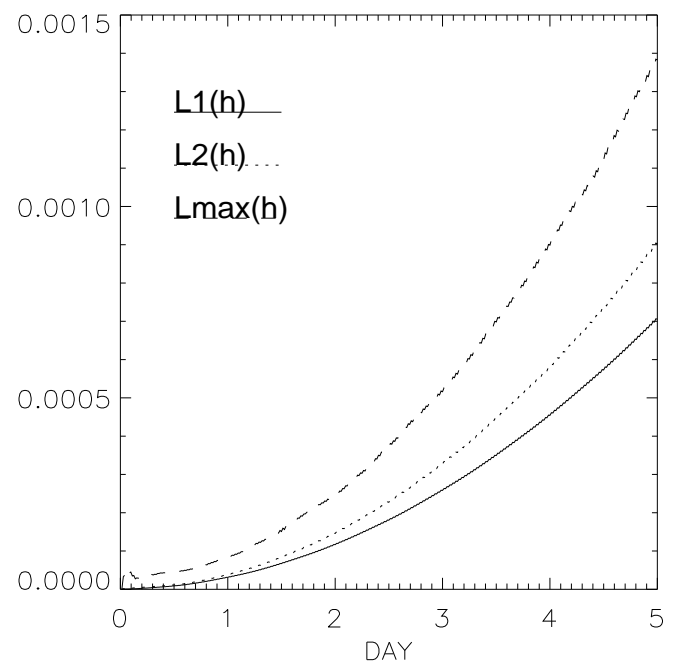

(b)

Fig. 3: Case 2 (a) Contour plot of the height field error for $\alpha=\pi / 2-0.05$ and $\Delta t=1200$ seconds. Contour interval is $\mathbf{0 . 0 5 m}$. (b) $l_{1}$ (solid), $l_{2}$ (short dash), and $l_{\infty}$ (long dash) errors.

Fig. 4b. As in the previous case, the errors are small and smooth, but monotonically increasing in time.

\subsection{ZONAL FLOW OVER AN ISOLATED MOUNTAIN}

This case does not admit an analytic solution. It consists of the zonal flow, defined in Case 2 , impinging on a mountain. The wind and height field are the same when $\alpha=0$. The equivalent depth $h_{0}$ is $5960 \mathrm{~m}$ for this case and the surface or mountain height is given by

$$
h_{s}=h_{s_{0}}(1-r / R)
$$

where $h_{s_{0}}=2000 \mathrm{~m}, R=\pi / 9$ and $r^{2}=\min \left[R^{2},\left(\lambda-\lambda_{c}\right)^{2}+\left(\theta-\theta_{c}\right)^{2}\right]$. The center is taken as $\lambda_{c}=3 \pi / 2$ and $\theta_{c}=\pi / 6$.

The representation of the mountain height and the varying depth of the fluid for this case are a challenge for all methods. Since the fluid is not in geostrophic balance at the initial time, a rapidly moving internal gravity wave is generated. Figure 5a shows the difference in height field and initial condition 1 day into the integration. At the opposite side of the sphere from the mountain the expansion wave has coalesced. 


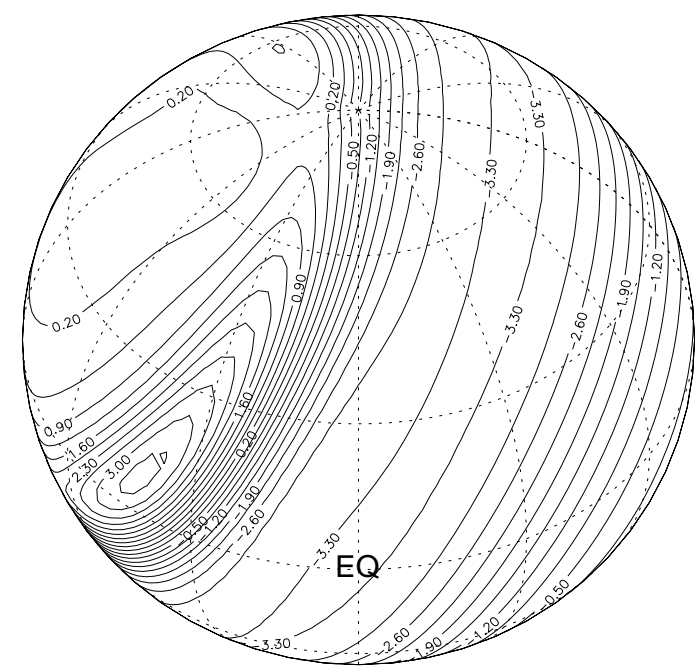

(a)

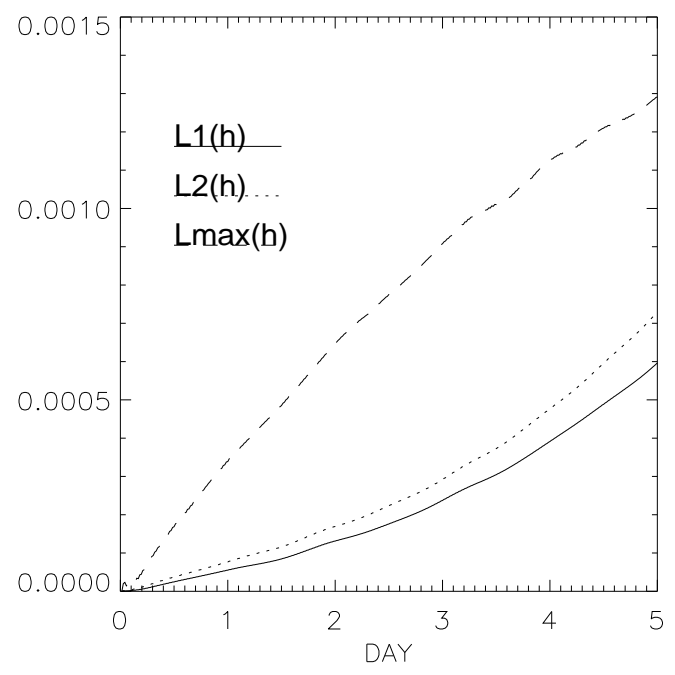

(b)

Fig. 4: Case 3 (a) Contour plot of the height field error after 5 days, for $\alpha=\pi / 2-0.05$ and $\Delta t=1200$ seconds; (b) $l_{1}$ (solid), $l_{2}$ (short dash), and $l_{\infty}$ (long dash) errors.

The height field is shown in Fig. 6a-d for the initial state, day 5, day 10, and day 15 respectively. These results are quite similar to the spectral Eulerian model results, i.e. a rapid evolution from a meridionally smooth zonally symmetric flow to an irregular high wavenumber state by day 15. Because the semi-Lagrangian interpolation smoothes the fields, the height field is without any spectral ringing or evidence of instability in the vicinity of the mountain. The location and magnitude of the major flow features are accurately captured and reflect the same features as the spectral T42 solution.

The change in global quantities of mass, enstrophy, and total energy from the initial conditions as a function of time are shown in Fig. 7. The total mass and enstrophy change very little over the integration. The total energy is increasing, with a total change of $1.3 \%$ by 15 days. The semi-Lagrangian scheme is not energy conserving, in the strict sense. This is a result of the advective formulation. But these errors are relatively small and no attempt has been made to compensate for mass or energy lost in the results presented. For application to climate modeling, where long time integrations are required, a fixer must be added to prevent imbalances from affecting the asymptotic states. 


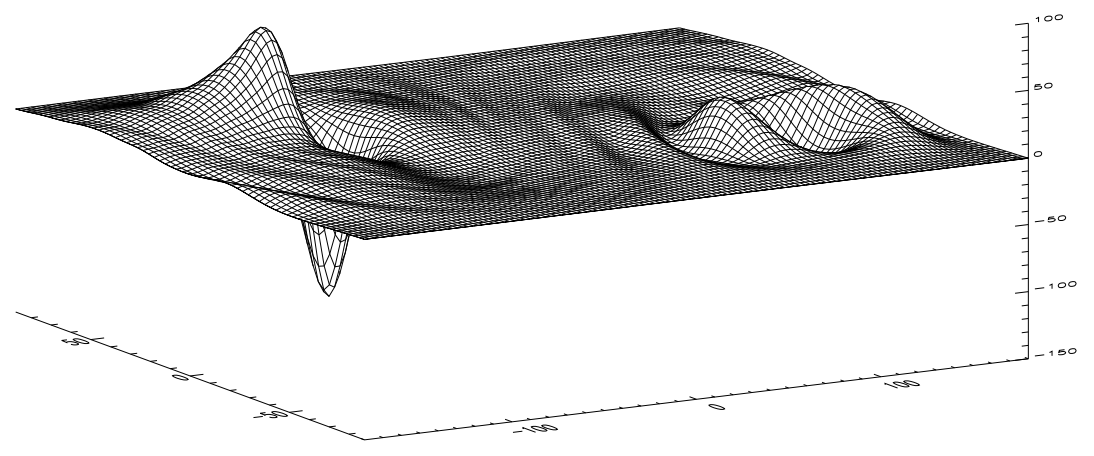

Fig. 5: Case 5 The difference in height field from initial condition after one day for $\Delta t=1200$ seconds.

It should also be noted that no added diffusion was necessary to control the tail of the energy spectra as was done in the Eulerian spectral method. Apparently enough diffusion is introduced by SLT interpolation.

\subsection{ROSSBY-HAURWITZ WAVE}

The last test case is a wavenumber four, Rossby-Haurwitz wave. Rossby-Haurwitz waves are analytic solutions to the barotropic vorticity equation. The relation to solutions of the nonlinear shallow water equations is not quite clear. For a stable wave pattern like wavenumber four, the test can indicate if there are other modes of systematic error that a scheme introduces. In Fig. $8 \mathrm{a}-\mathrm{d}$, the basic wave pattern is well maintained through the entire simulation. The spectral Eulerian solution requires introduction of artificial dissipation (for example a biharmonic term) to control the tail of the energy spectrum. The semi-Lagrangian method apparently has no need for this as enough dissipation is introduced by the method.

The change in global quantities of mass, enstrophy, and total energy from the initial conditions as a function of time are showed in Fig. 9. For this case, the total mass almost has no change, but the enstrophy and the total energy is decreasing (eventually), with a total change of $-1.2 \%$ by 15 days. 

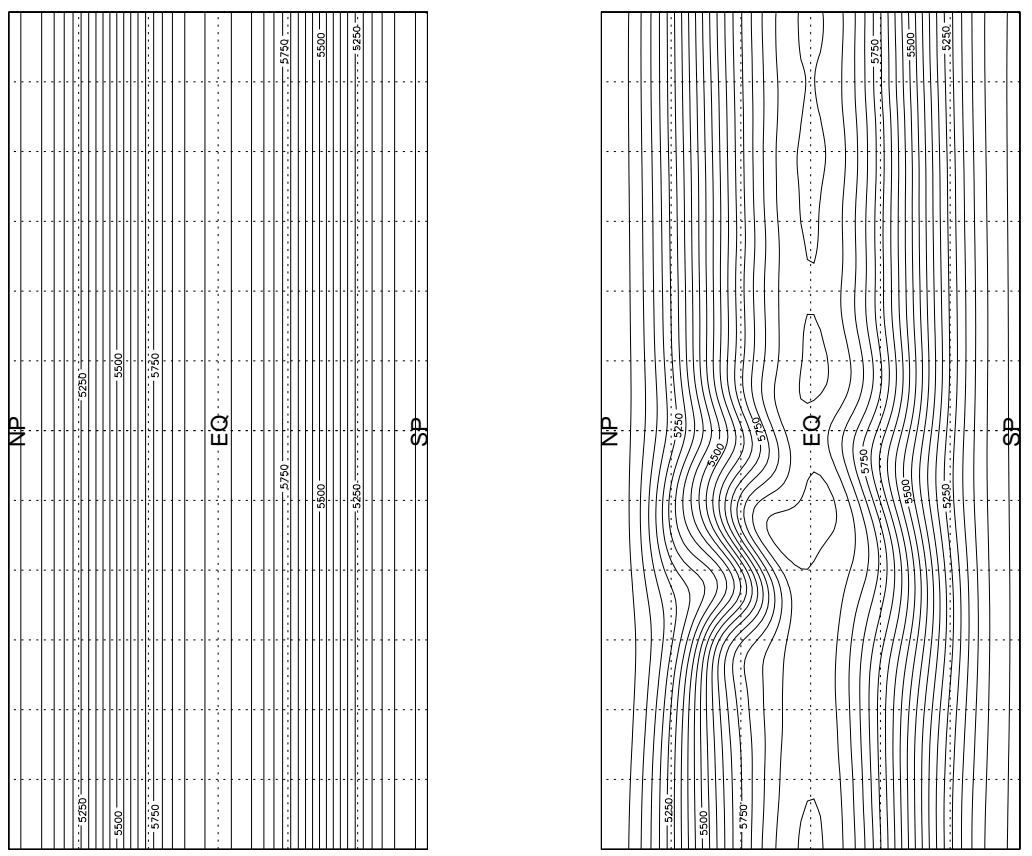

(a)

(b)
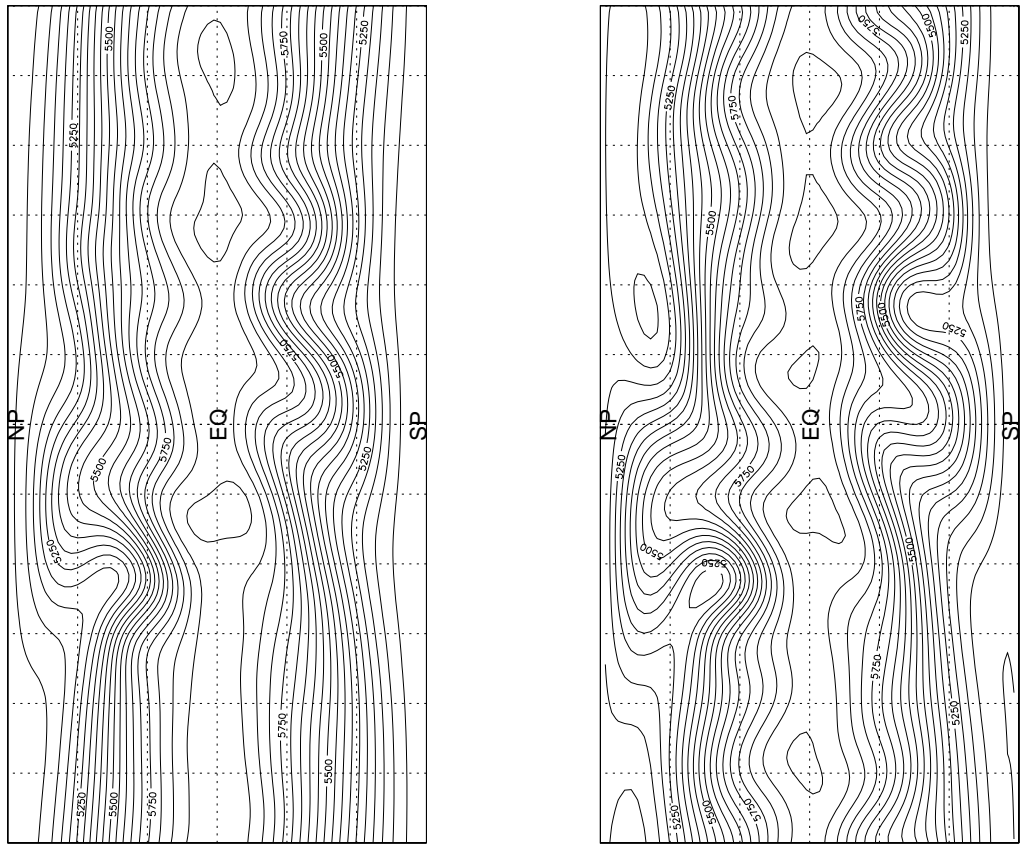

(c)

(d)

Fig. 6: Case 5 (cont.) Contour plots of the height field for $\Delta t=1200$ seconds at (a) day 0 , (b) day 5, (c) day 10, and (d) day 15 on a cylindrical equidistant projection. Contour interval is $50 \mathrm{~m}$. 


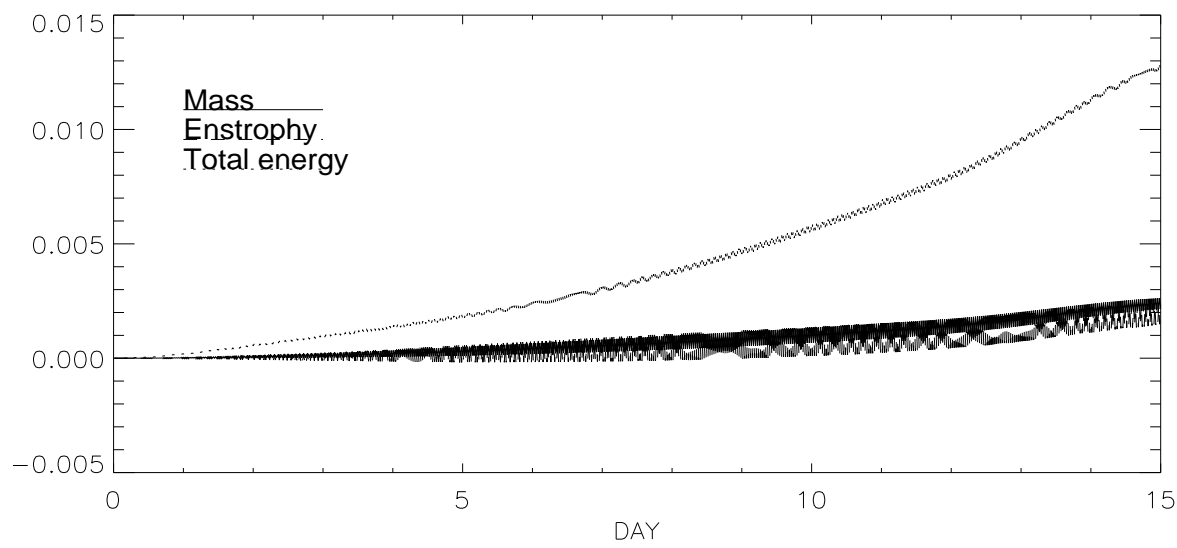

Fig. 7: Case 5 (cont.) Relative change of global mass (solid), relative change of total energy (short dash), and enstrophy (long dash). 

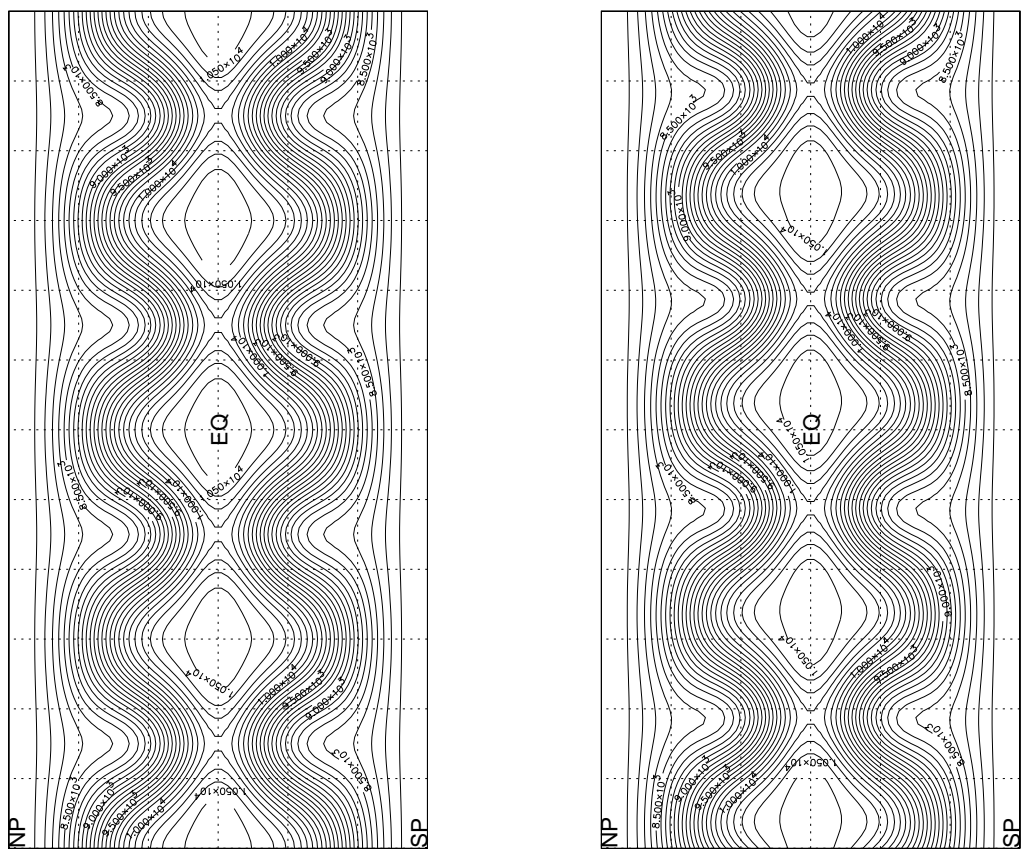

(a)

(b)
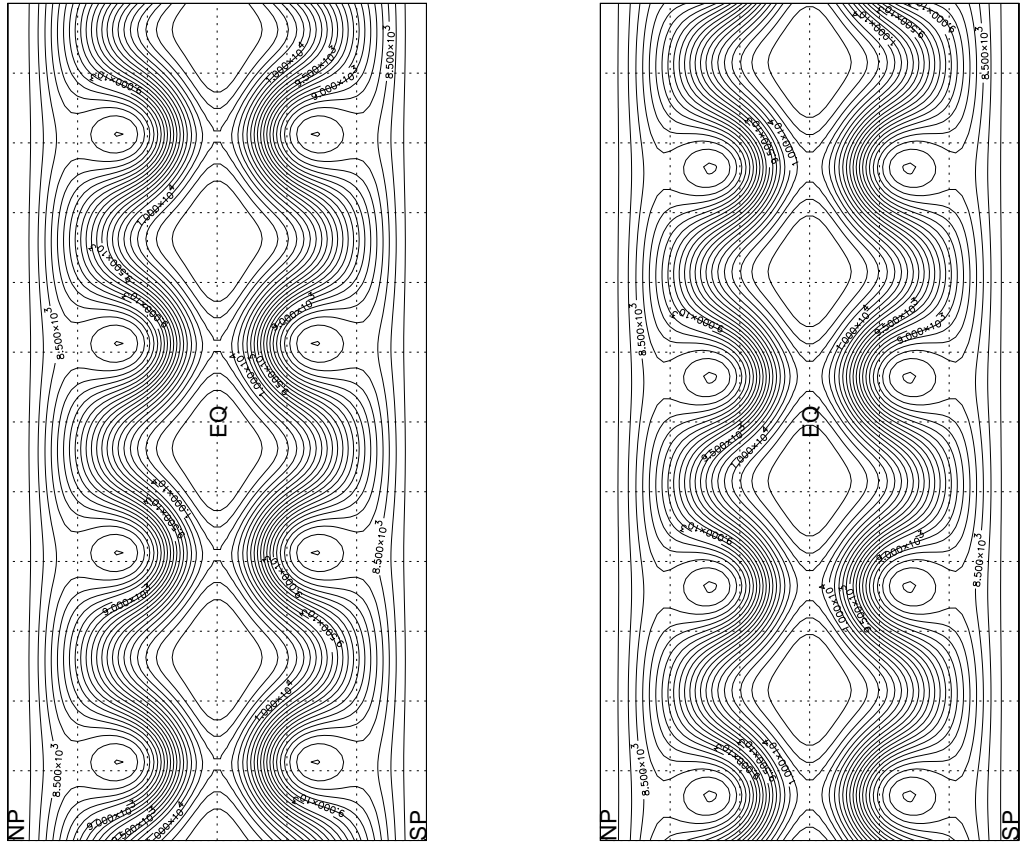

(c)

(d)

Fig. 8: Case 6 Contour plots of the height field for $\Delta t=1200$ seconds at (a) day 0, (b) day $1,(\mathrm{c})$ day 7 , and (d) day 14. 


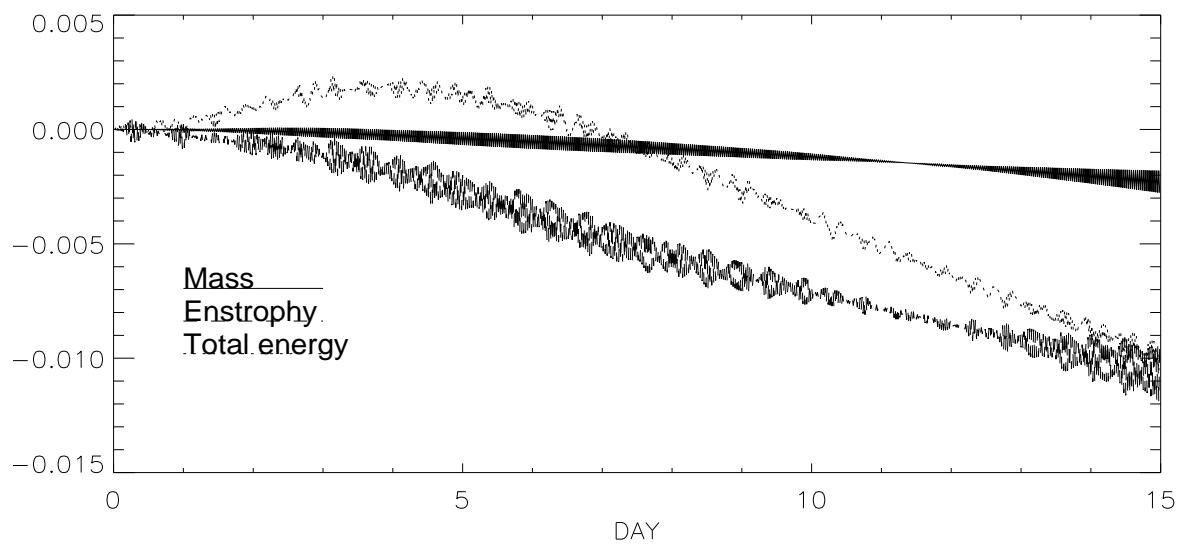

Fig. 9: Case 6 (cont.) Change in global mass (solid), total energy (short dash), and enstrophy (long dash). 


\section{CONCLUSIONS}

The semi-Lagrangian transport algorithm is an important component of global atmospheric circulation models, and will continue to be attractive for the next generation of models because of its accuracy and ability to take long timesteps. A new advective formulation of the shallow water equations in a spherical geometry allows a natural application of the SLT algorithm. The method presented here combines semi-Lagrangian transport with a spectral, semi-implicit algorithm. The results compare favorably in terms of accuracy, stability and smoothness of solution with a spectral Eulerian shallow water equation model. Future work will extend this formulation to the full baroclinic equations and explore solutions using two time-level semiLagrangian methods as well as other gridding systems. 


\section{References}

[1] Bates, J. R., F. H. M. Semazzi, R. W. Higgins, S. R. M. Barros 1990. Integration of the Shallow Water Equations on the Sphere using a Vector Semi-Lagrangian Scheme with a Multigrid Solver, MWR, vol 118, pp. 1615-1627.

[2] Hack, J. J. and R. Jakob 1992. Description of a global shallow water model based on the spectral transfor method, NCAR Tech. Note NCAR/TN-343+STR, National Center for Atmospheric Research, Boulder, Colo.

[3] Jakob, R., J. J. Hack and D. L. Williamson 1994. Spectral Transform Solutions to the Shallow Water Test Set NCAR/0301/94-04.

[4] Ritchie, H. 1985. Application of the semi-Lagrangian Integration Scheme in a Regional Forecast Model, MWR, vol 113, pp. 424-435.

[5] Ritchie, H. 1988. Application of the semi-Lagrangian method to a Spectral Model fo the Shallow Water Equations, MWR, vol 116, pp. 1587-1598.

[6] Robert, A., J. Henderson and C. Turnbull 1972. An Implicit Time Integration Scheme for Baroclinic Models of the Atmosphere, MWR, vol 100, pp. 329-335.

[7] Serrin, J. 1959. Mathematical Principles of Classical Fluid Mechanics, Encyclopedia of Physics, Vol. 13, 1, Springer, Berlin.

[8] Staniforth, A. and J. Côté 1991. Semi-Lagrangian Integration Schemes for Atmospheric Models - A Review, MWR, vol 119, pp. 2206-2223.

[9] Washington, W. M. and C. L. Parkinson 1988. An Introduction to Three-Dimensional Climate Modeling, University Science Books.

[10] Williamson, D. L. and P. J. Rasch 1989. Two-Dimensional Semi-Lagrangian Transport with Shape-Preserving Interpolation, MWR, vol 117, No. 1, pp. 102-128.

[11] Williamson, D. L., J. B. Drake, J. J. Hack, R. Jakob, and P. N. Swarztrauber 1992. A standard test set for numerical approximations to the shallow water equations in sperical geometry, J. Comp. Phys., 102, pp. 211-224.

[12] Williamson, D. L. and J. G. Olson 1994. Climate Simulations with a Semi-Lagrangian Version of the NCAR Community Climate Model, MWR, vol 122, pp. 1594-1610. 
ORNL/TM-2001/216

\section{INTERNAL DISTRIBUTION}

1. J. B. Drake

2. T. Zacharia

3. Central Research Library
4. ORNL Laboratory Records-RC

5-6. ORNL Laboratory Records-OSTI

\section{EXTERNAL DISTRIBUTION}

7. Daniel X. Guo

Department of Mathematics and Statistics

University of North Carolina at Wilmington

Wilmington, NC 94550

8. Dr. David C. Bader

Environmental Sciences Division, SC-74

Department of Energy

19901 Germantown, Rd.

Germantown, MD 20874-1290 